\title{
A Review of Image Binarization Techniques for Ancient Degraded Documents
}

\author{
Punam A. Mahajan ${ }^{1}$, Swati A. Patil ${ }^{2}$ \\ ${ }^{1,2}$ G.H. Raisoni Institute of Engineering and Management, Jalgaon
}

\begin{abstract}
Image Binarization Technique depends on the segmentation technique which is major technique for separation of pixel values such as black as foreground and white as background. Segmentation of the degraded document images can be done by using image binarization techniques in order to acquire a clear image which will be visible means which is easy to understand them. Thresholding technique and Contrast image construction are the important techniques for degraded document image binarization. For this document binarization technique, many binarization techniques has been proposed.
\end{abstract}

Keywords: Recursive Thresholding, Gabor Filter, Contrast Image Construction, Contour Based Image Segmentation

\section{Introduction}

Document images may be degraded due to the poor quality of paper, ink plot, fading, document aging. Visibility of document degrades due to the scanning and printing that means it is very difficult to understand them. Degraded document image restoration plays a very important role in enhancing the degraded noise in images. Document Image Binarization is the major technique for the segmentation [1] of the text values from the background images. Separation of the pixel values into black as foreground and white as background can be acquire to extract the clear image.

In document binarization process, thresholding technique is well-known technique. Local and Global Thresholding are two important types of the thresholding process. Global thresholding [5] has good performance because there is a good separation between the foreground and the background. On the other hand, local area information may guide the threshold value for each pixel in local (adaptive) thresholding techniques [5]. These techniques have been widely used in document image analysis because they have a better performance in extracting the character strokes from an image that contains spatially uneven gray levels due to degradations.

To enhance the degraded document, its image is binarized before processing it. It is nothing but segmenting the document background \& the foreground text. For the document image processing task the perfect document image binarization technique is the best option. After many types of work done in the binarization technique, the thresholding of degraded document images is still found to be a challenging task because of the high inter/intra variation between the text stroke and the document background across various document images. The stroke brightness, document background, stroke width and stroke connection vary in the handwritten text within the degraded documents [1].

\section{Literature Survey}

Many thresholding techniques [1] have been reported for document image binarization. Adaptive thresholding [13] estimates a local threshold for each document image pixel and it is often a better approach to deal with different variations within degraded document images. the early window-based adaptive thresholding techniques [14], [15] estimate the local threshold by using the mean and the standard variation of image pixels within a local neighbourhood window. The thresholding performance depends heavily on the window size and hence the character stroke width and this is the main drawback of window-based thresholding technique. Other approaches have also been reported, including background subtraction, texture analysis, recursive method, decomposition method, contour completion, Markov Random Field, matched wavelet, cross section sequence graph analysis, self-learning, Laplacian energy user assistance and combination of Binarization techniques. These methods combine different types of image information and domain knowledge and are often complex. The local image contrast and the local image gradient are very useful features for segmenting the text from the document background because the document text usually has certain image contrast to the neighbouring document background. They are very effective and have been used in many document image binarization techniques. In Bernsen's Technique, the local contrast is defined as follows:

$C(i, j)=\operatorname{Imax}(i, j)-\operatorname{Imin}(i, j)$

where $C(i, j)$ denotes the contrast of an image pixel $(i, j$ ), $I \max (i, j)$ and $I \min (i, j)$ denote the maximum and minimum intensities within a local neighbourhood windows of $(i, j)$, respectively. If the local contrast $C(i, j)$ is smaller than athreshold, the pixel is set as background directly. Otherwiseit will be classified into text or background by comparing with the mean of $\operatorname{Imax}(i, j)$ and $\operatorname{Imin}(i, j)$. Bernsen's method is simple, but cannot work properly on degraded document images with a complex document background.

\section{Techniques}

\subsection{Global and Local Thresholding Technique}

According to the global threshold value set, the pixels of image separated into the background or text. This technique is very simple and fast. If the background noise is unevenly 


\section{International Journal of Science and Research (IJSR) \\ ISSN (Online): 2319-7064}

Index Copernicus Value (2013): 6.14 | Impact Factor (2014): 5.611

distributed in the entire image, then they cannot be easily adapted.

Local threshold technique is more adaptive where the pixels of the image are classified into text or background according to the local threshold determined by neighbouring pixels. This technique can deal with the different kinds of noise existing in one image. Local threshold for each pixel is calculated using Niblack method which is depend on the local mean value and the local standard deviation in the neighbouring of the pixels.

\subsection{Contrast Image Construction and Text Stroke Edge Pixel Detection}

Document binarization technique issues can be overcomed by using adaptive image contrast technique. Local image contrast and the local image gradient are combination refers to the adaptive image contrast. This technique causes tolerant to the text and background variation caused by different types of document degradations.

Local image contrast and the local image gradient both the techniques are useful where the segmentation of text from the background is essential. For edge detection and to detect text stroke edges of document image effectively, image gradient feature has been widely used. To supress the backgroung variations, local contrast evaluated by the local image maximum and minimum.

\subsection{Recursive Thresholding Technique}

A Recursive Thresholding method for Image Segmentation was performed by extending the Otsu's method. This method is based on discriminant analysis and the threshold operation. This method is considered as the dividing the pixels of an image into foreground and background. The new approach had implemented in the range of document images, especially real-life bank checks. The recursive method is developed without any restrictions on the number of objects in the digital image. This approach extracts the brightest homogeneous object from an input image at each recursion, and eliminates the darkest homogeneous object after the last recursion.

\subsection{Preprocessing Using Gabor Filter}

Gabor Transformation includes: Standard deviation of Gaussian distribution(Orientation and sigma).The value of sigma is tradeoff larger values. These sigma values are more robust to noise but it creates spurious rings. On the other hand, Smaller values removes noise and it produces less spurious rings. The steps of applying gabor filter in the preprocessing phase are such as 1. Firstly take an input image 2.apply gabor filter 3. Transform the values of intensity image to enhance the contrast of the image 4.Enhance contrast of the small regions of image 5 . Subtraction between input image and contrasted image can be done. 6 . Finally compliment of the result calculated.

\subsection{Contour Based Image Segmentation Technique}

Object extractions play a major role in the contour based image segmentation. In this technique, Morphological edge dectector can be implemented with region growing techniques. The first step includes is image enhancement using morphological closing operation and detection process is carried out by morphological dilation using edge detector. The implementation of morphological operator has been carried out in order to enhance the image over the edges. In addition to this, Region growing and region merging Technique also has been implemented for detecting accurate edge extraction and to overcome image segmentation problems. Here, the inputimage is operated to a morphological closing operation and edge detector then implemented region growing with region merging techniques in order to implement the image segmentation. This segmentation can be implemented only in theimage then processed by using morphological operation in order to get a clear contour of the image.

\subsection{Otsu threshold and an Empirical technique for thresholding selection}

Otsu's thresholding technique is used for automatic binarization level decision, based on the shape of the histogram. The image is composed of two basic classes: Foreground and Background. It then computes an optimal threshold value that minimizes the weighted within class variances of these two classes in which it minimizes the class variance to determine the binarization level. It automatically performs the clustering-based image thresholding, or, the reduction of a gray level image to a binary image.

\subsection{Canny Edge Detector}

The Canny edge detector is effectively an edg detection operator which uses a multi stage algorithm to detect a wide range of edges in images. The main aim of this algorithm was to discover the optimal edge detection algorithm. In this situation, an "optimal" edge detector means: (1) real edges in the image as possible.(2)good localization - edges marked should be as close as possible to the edge in the real image. (3) minimal response - a given edge in the image should only be marked once, and where possible, image noise should not create false edges. The Canny Edge detection algorithm runs in 4separate steps: (1) Smoothing: Blurring of the image to remove noise. (2) Finding gradients: The edges should be marked where the gradients of the image has large magnitudes. (3) Non-maximum suppression: Only local maxima should be marked as edges. (4)Double thresholding: Potential edges are determined by thresholding. Final edges are determined by suppressing all edges that are not connected to a very certain (strong) edge.

\section{Performance Factors}

\subsection{Niblack's Method}

Niblack's method produces great amount of background noise. This method is suitable for almost all types of images, but during the processing stage, it does not able to remove the unimportant information. This method implements the 


\section{International Journal of Science and Research (IJSR) \\ ISSN (Online): 2319-7064 \\ Index Copernicus Value (2013): 6.14 | Impact Factor (2014): 5.611}

sliding rectangular window over a grayscale image to calculate threshold for each pixel. The threshold calculation involves evaluation of the local mean and standard deviation of the each pixel present inside the window.

\subsection{Kim's Method}

Kim's method suffers from variable strength of characters but has good result in maximum input document images. This method proposes a local adaptive thresholding method where an image is regarded as a $3 \mathrm{D}$ terrain. The local property of this model is characterized by a water flow model. The water flow model locally detects the valleys corresponding to regions that are lower than neighbouring regions.

\subsection{Sauvola's Method}

Sauvola's method overcomes the background noise problems but produces thinned and broken characters. This method is an improvement of Niblacks method. This method solves the problem of presence of large amount of noise in the background areas. Threshold value can be computed by using the dynamic range of images gray-scale standard deviation. However, this method has some problems also, when the text pixel value close to the foreground image.

\subsection{Nick Method}

Nick method is an improvement of niblack method where it works very well for most degraded document. This method solves the problem of noise in white pages and low contrast problem.

\section{References}

[1] Graham Leedham, Chen Yan, KalyanTakru, Joie HadiNata Tan and Li Mian,"Comparison of Some Thresholding Algorithms for Text/Background Segmentation in Difficult Document Images", Proc. of the Seventh International Conference on Document Analysis and Recognition, 2003.

[2] M. Sezgin and B. Sankur, "Survey over image thresholding techniques and quantitative performance evaluation," J. Electron.Imag., vol. 13, no. 1, pp. 146165, Jan. 2004.

[3] K. Kaviya Selvi and R. S. Sabeensisn, "Restoration of Degraded Documents Using Image binarization Technique", ARPN Journal of Engineering and Applied sciences, Vol. 10, no. 7, April 2015.

[4] Sayali Shukla, Ashwini Sonawane, Pooja Tiwari, "Improving Degraded Document Images Using Binarization Technique," International Journal of Scientific and Technology Research, Vol. 3, Issue 5, May 2014

[5] Vishnupriya Raj and C. Arunkumar, "Content Restoration of Degraded Termitte Bitten Document Images," International Journal of Engineering Research and Applications, Vol. 4, Issue 5, May 2014.

[6] S. Geman and D. Geman, "Stochastic Relaxation Gibb's Distribution and the Bayesian Restoration of Images," IEEE transaction on Pattern Analysis and Machine Intelligence, 6(6), 1984.
[7] B. Gatos, I. Pratikakis, and S. J. Perantonis, "Adaptive Degraded document image binarization," Elsevier Trans. Pattern Recogn., Vol. 39, pp. 317-327, 2006

[8] Md. Iqbal QuraishiDe, Krishna Gopal Dhal,and Saheb Mondal, Goutam Das, "A novel hybrid approach to restore historical degraded documentsISSP , "vol. 1,pp. 185-189, IEEE 2013.

[9] J.-D. Yang, Y.-S. Chen and W.-H. Hsu, "Adaptive thresholding algorithm and its hardware implementation," Elsevier Trans. Pattern Recogn., Vol. 15, no. 2, pp. 141-150, 1994.

[10] I. Pratikakis, B. Gatos, and K. Ntirogiannis, "ICFHR 1012 competition on handwritten document image binarization," in Proc ICFHR, Sep. 2012, pp. 813-818.

[11] R. F. Moghaddam and M. Cheriest, " Rsldi: Restoration of Single-sided low-quality document images," Pattern Recognition, vol. 42,pp. 3355-3364,January 2009.

[12] O. Trier and T.Text, "Evaluation of Binarization methods for document images," IEEE Trans. Analysis and Machine Intelligence, Vol. 17, pp. 312-315, March 1995.

[13] J. Bernsen, "Dynamic thresholding of gray-level images," in Proc. Int. Conf. Pattern Recognition, Oct 1986, pp.1251-1255.

[14] J. Sauvola and M. Pietikainen, "Adaptive document image binarization," Pattern Recognition, vol.33, no. 2, pp.225-236, 2000.

[15] W. Niblack, "An Introduction to Digital Image Processing,” Englewood Cliffs, NJ:Prentice-Hall,1986. 